Original article

\title{
Computational comparison of conventional and novel electroconvulsive therapy electrode placements for the treatment of depression
}

\author{
Siwei Bai ${ }^{\mathrm{a}, \mathrm{b}, \mathrm{c}}$, Donel Martin ${ }^{\mathrm{d}, \mathrm{e}}$, Tianruo Guo ${ }^{\mathrm{c}}$, Socrates Dokos ${ }^{\mathrm{c}}$, Colleen Loo ${ }^{\mathrm{d}, \mathrm{e}, \mathrm{f}, *}$ \\ a Munich School of Bioengineering, Technical University of Munich (TUM), 85748 Garching, Germany \\ ${ }^{\mathrm{b}}$ Department of Electrical and Computer Engineering, TUM, 80333 Munich, Germany \\ ${ }^{\mathrm{c}}$ Graduate School of Biomedical Engineering, Faculty of Engineering, University of New South Wales (UNSW), NSW 2052, Australia \\ d School of Psychiatry, UNSW, NSW 2052, Australia \\ e Black Dog Institute, NSW 2031, Australia \\ f Department of Psychiatry, St George Hospital, NSW 2217, Australia
}

\section{A R T I C L E I N F O}

\section{Article history:}

Received 18 March 2019

Received in revised form 17 May 2019

Accepted 18 May 2019

Available online 21 June 2019

\section{Keywords:}

Electroconvulsive therapy (ECT)

Brain stimulation

Electrode placement

Computational model

Efficacy

Depression

\begin{abstract}
A B S T R A C T
Background: Electroconvulsive therapy (ECT) is a highly effective treatment for severe psychiatric disorders. Despite its high efficacy, the use of ECT would be greater if the risk of cognitive side effects were reduced. Over the last 20 years, developments in ECT technique, including improvements in the dosing methodology and modification of the stimulus waveform, have allowed for improved treatment methods with reduced adverse cognitive effects. There is increasing evidence that the electrode placement is important for orienting the electrical stimulus and therefore modifying treatment outcomes, with potential for further improvement of the placements currently used in ECT.

Objective: We used computational modelling to perform an in-depth examination into regional differences in brain excitation by the ECT stimulus for several lesser known and novel electrode placements, in order to investigate the potential for an electrode placement that may optimise clinical outcomes.

Methods: High resolution finite element human head models were generated from MRI scans of three subjects. The models were used to compare regional differences in average electric field (EF) magnitude among a total of thirteen bipolar ECT electrode placements, i.e. three conventional placements as well as ten lesser known and novel placements.

Results and conclusion: In this exploratory study on a systemic comparison of thirteen ECT electrode placements, the EF magnitude at regions of interest (ROIs) was highly dependent upon the position of both electrodes, especially the ROIs close to the cortical surface. Compared to conventional right-unilateral (RUL) ECT using a temporo-parietal placement, fronto-parietal and supraorbito-parietal RUL also robustly stimulated brain regions considered important for efficacy, while sparing regions related to cognitive functions, and may be a preferrable approach to the currently used placement for RUL ECT. The simulations also found that regional average EF magnitude varied between individual subjects, due to factors such as head size, and results also depended on the size of the defined ROI.
\end{abstract}

(c) 2019 Elsevier Masson SAS. All rights reserved.

\section{Introduction}

Three main electrode placements are used in conventional electroconvulsive therapy (ECT): bitemporal (BT), bifrontal (BF)

\footnotetext{
* Corresponding author at: Black Dog Institute, Hospital Road, Prince of Wales Hospital, Randwick, NSW 2031, Australia.

E-mail addresses: siwei.bai@tum.de (S. Bai), donel.martin@unsw.edu.au (D. Martin), t.guo@unsw.edu.au (T. Guo),s.dokos@unsw.edu.au (S. Dokos), colleen.loo@unsw.edu.au (C. Loo).
}

and right unilateral (RUL). Among these, BT represents the original form of ECT, and has generally been found to have the fastest speed of response [1]. However, BT ECT has also been shown to cause greater cognitive side-effects $[2,3]$. The RUL electrode placement, initially proposed by d'Elia [4], represents one of the most commonly applied placements in contemporary ECT due its superior cognitive side-effect profile [3,5]. The antidepressant effect of RUL ECT is also dose sensitive, with low dose RUL treatment found to be less effective [6,3]. The BF electrode placement was initially suggested by Abrams et al $[7,8]$. Recent studies have shown that BF ECT has antidepressant outcomes 
comparable to BT ECT, and may also have a relatively low risk of cognitive side-effects [9-13], though one randomized control trial failed to find any cognitive advantage with BF compared to both BT and RUL placements [1]. Thus, while each of these conventional placements have demonstrated therapeutic efficacy, the clinical effects mainly differ with regards to speed of therapeutic response and the magnitude of cognitive side-effects.

Recent computational modelling studies have demonstrated that the distribution and spatial extent of brain regions directly stimulated by the electrical current differ as ECT placement is varied [14-20]. These simulation results concur with those of neuroimaging studies performed after ECT [21]. Combined with evidence from clinical trials, this suggests that clinical and cognitive outcomes are associated with the topographical distribution of the ECT current (which in turn is contingent on the position of the stimulating electrodes). Other evidence also supports the importance of the direct effects of the ECT stimulus itself, rather than the subsequent induced seizure, for the efficacy of ECT. For example, it is possible to give forms of ECT (e.g. RUL ECT dosed at the patient's seizure threshold) which involve a seizure but have relatively low efficacy $[2,5,22]$. A recent proof of concept study further showed that BF ECT given at a subconvulsive dose had antidepressant effects [23]. Therefore, it is possible to further optimise current ECT treatment approaches by investigating direct stimulation effects of different electrode placements using computational models.

Novel electrode placements have been proposed, most notably including the bilateral "left anterior right temporal" (LART) placement [24], fronto-parietal placement for RUL ECT [25] and focal electrically administered seizure therapy (FEAST), a novel approach to RUL ECT using asymmetrical electrodes [26]. Swartz and colleagues in their open trials and double-blind pilot study reported significant antidepressant efficacy for LART, which was comparable to BT, with less cognitive side-effects $[24,27,28]$. The fronto-parietal RUL placement was first studied in controlled trials in the 1970s where the cognitive side-effects were compared to those of standard temporo-parietal RUL placement, although no significant advantage was found [29,30]. This placement was revisited recently in a case report which compared cognitive outcomes between fronto-parietal and temporo-parietal RUL, finding an overall advantage with fronto-parietal RUL [25]. Most recently, open label pilot studies of FEAST suggest that it may have comparable efficacy to conventional temporo-parietal RUL ECT, with minimal cognitive side effects [31,32]. These are examples of manipulations of electrode placement to optimise clinical outcomes, with effects demonstrated in small clinical trials. Using computational modelling allows the investigation of multiple variations of electrode placement, with estimates of electric field (EF) strengths in key brain regions.

To date, it remains unclear which brain regions should be maximally targeted for therapeutic effects. Computational modelling of the ECT stimulus using conventional electrode placements suggests that in addition to causing broad stimulation of frontal and temporal regions, conventional placements result in direct stimulation of frontal midline structures [18]. Of these, the subgenual anterior cingulate cortex (SAC) in particular, as well as its immediate surrounding structures, has been suggested to have a key role in the pathophysiology and treatment of depression [33,34]. Indeed, focalised stimulation of the SAC using deep brain stimulation has been shown to cause antidepressant effects [35]. Following a course of ECT, changes in SAC theta activity have been found to be associated with reduced psychotic symptoms in MDD patients [36]. Together, these converging findings suggest that maximising stimulation of the SAC may be important for antidepressant efficacy.
Memory related side-effects are the most prominent cognitive outcomes [37,38], and are generally considered to be associated with direct stimulation of medial temporal regions [39]. However, as conventional ECT placements stimulate both frontal and temporal regions $[15-18,20]$, it remains unclear which specific memory-related regions are associated with particular sideeffects. While the hippocampus is widely recognised as critical to memory functions, including encoding, retrieval and working memory [40], frontal regions, including the inferior and middle frontal gyri, are also important for memory retrieval [41,42]. Hemispheric dominance additionally plays an important role, with the left hemisphere known to be dominant for language and verbal processing in most people $[43,44]$, and hence more critical for side-effects involving verbal processing (e.g. verbal anterograde and retrograde memory). Electrode placements which are associated with reduced stimulation of left-sided memory based regions (i.e. hippocampus, inferior and medial frontal gyri) could then be associated with lesser cognitive side-effects.

In this study, we therefore used computational modelling to perform an in-depth examination into regional differences in brain excitation by the ECT stimulus for different electrode placements. We hypothesised that greater stimulation of the SAC region would be associated with greater treatment efficacy, whilst conversely reduced stimulation of left-sided memory based regions would be associated with reduced cognitive side-effects. In addition to examining stimulation effects from conventional and other lesser known placements, several novel placements were examined which were designed for optimal stimulation of the SAC region. Stimulation effects were investigated in head models derived from MRI scans of several different subjects to minimise the effects of inter-individual differences (i.e. head size).

\section{Methods}

\subsection{Computational head model development}

Head models of three subjects (SUB1-3) were reconstructed from their T1-weighted 3T MRI head scans. Detailed methods on model formulation can be found in our previous studies [45,18]. A total of thirteen bipolar ECT electrode placements were simulated. In each placement, an electrical current of $800 \mathrm{~mA}$ was delivered through the scalp over a pair of circular electrodes with a radius of $2.5 \mathrm{~cm}$. These current amplitudes represent typical levels of clinical ECT currents utilised. The electrode placements for these placements are described as follows, as illustrated in Figs. 1 \& 2 :

- BT: the centre of each electrode was placed on each side of the scalp $3 \mathrm{~cm}$ superior to the midpoint of a line connecting the external ear canal with the lateral canthus of the eye.

- BF: the centre of each electrode was placed $5 \mathrm{~cm}$ superior to the lateral canthus of each eye.

- temporo-parietal-unilateral: the anterior electrode was placed on the temporal position (described in BT placement) on the right side of the scalp, and the posterior was placed just right of the vertex of the head. This is the most common RUL placement.

- temporo-parietal-bilateral: the anterior electrode was placed at the same position as the temporo-parietal-unilateral placement, and the posterior electrode was placed just left of the vertex of the head.

- supraorbito-parietal-unilateral: the anterior electrode was placed above the supraorbital ridge of the right eye, and the posterior was placed just right of the vertex of the head.

- supraorbito-parietal-bilateral: the anterior electrode was placed at the same position as the supraorbito-parietal-unilateral placement, and the posterior electrode was placed just left of the vertex of the head. 


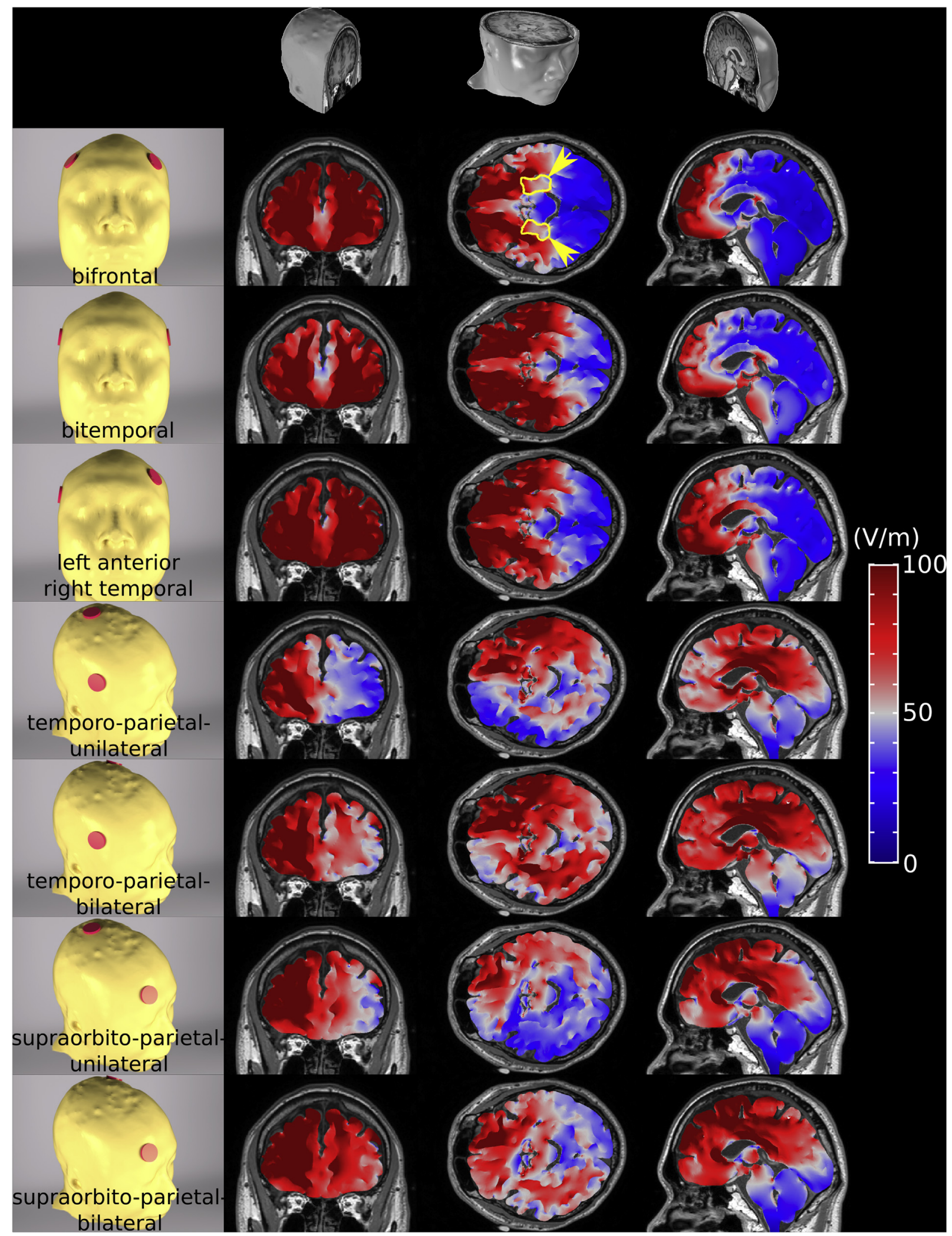

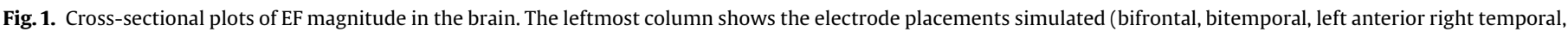

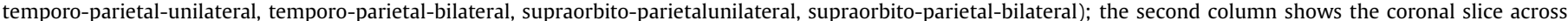

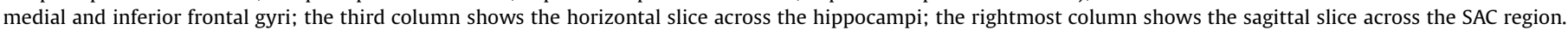

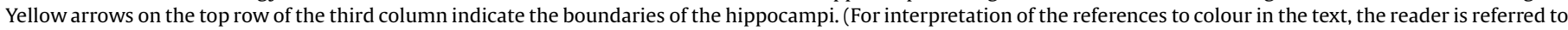
the web version of this article.) 


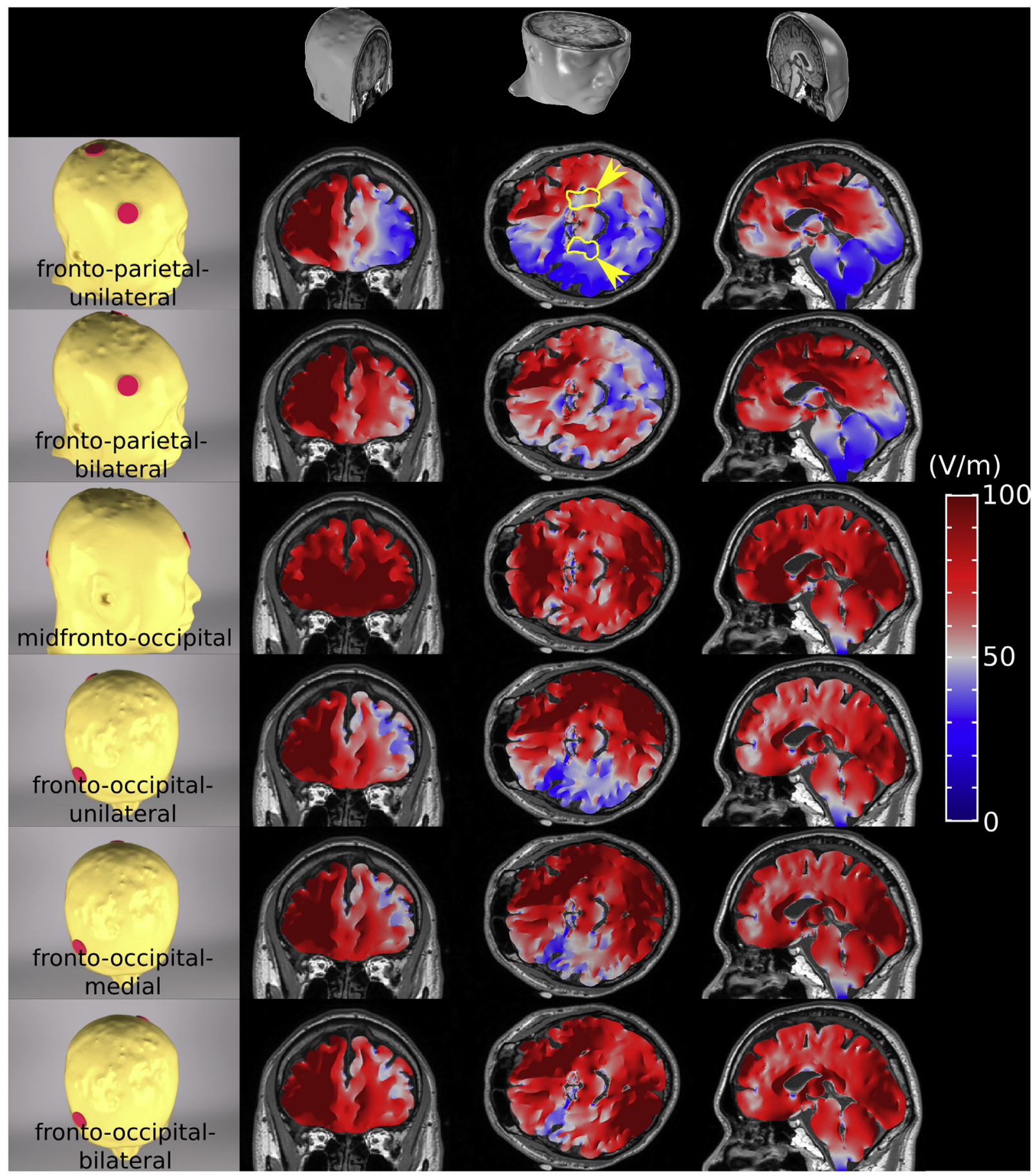

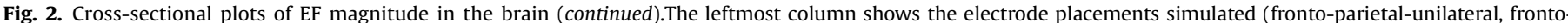

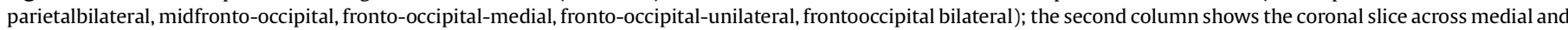

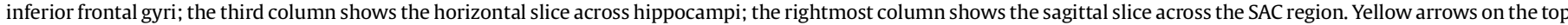

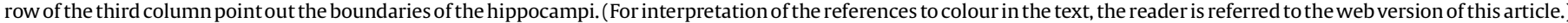

- midfronto-occipital: the anterior electrode was placed just above the nasion, and the posterior was placed just over the occiput.

- LART: the left electrode was placed at the same location as in BF, and the right electrode was at the same location as in BT.

- fronto-parietal-unilateral: the anterior electrode was placed on the frontal position (described in BF placement) on the right side of the scalp, and the posterior electrode was placed just right of the vertex of the head.

- fronto-parietal-bilateral: the anterior electrode was placed at the same position as in the fronto-parietal-unilateral placement, and the posterior electrode was placed just left of the vertex of the head.

- fronto-occipital-medial: the anterior electrode was placed in the frontal position (described in BF placement) on the right side of the scalp, and the posterior electrode was placed just over the occiput. This placement was inspired by the tDCS placements SO-OCC [45] and F-O [46].

- fronto-occipital-unilateral: the anterior electrode was placed at the same position as the fronto-occipital-medial placement, and the posterior electrode was placed just right of the occiput. 
- fronto-occipital-bilateral: the anterior electrode was placed at the same position as the fronto-occipital-medial placement, and the posterior electrode was placed just left of the occiput.

\subsection{Data analysis}

All simulations were carried out using the COMSOL Multiphysics v5.0 (COMSOL AB, Sweden) finite-element software package running on a Windows 64-bit Precision workstation (Dell, TX) with 24 GB RAM. To solve the stationary equations, a direct linear solver was utilised with an absolute error tolerance set to $10^{-5}$. It took $30 \mathrm{~min}$ to solve for each simulation, each with approximately $5 \times 10^{6}$ degrees of freedom.

Simulation results were analysed in MATLAB R2014b (MathWorks, MA) by comparing the average EF magnitude $\mathrm{E}$ in several ROIs in the brain, calculated using:

$\bar{E}=\frac{\int_{V}|E| d V}{\int_{V} d V}$

where $|E|$ is the average EF magnitude in the ROI in question, and $\int_{V}$ is the volume integral over this region. Note that the denominator is simply the volume of the ROI. Analysis of results was based on the "quasi-uniform" assumption, namely that the degree of activation in a target region is proportional to the local EF magnitude [47].

\section{Results}

The EF magnitude on slices across the key ROIs of this study is illustrated in Figs. 1 \& 2 for all electrode placements, and the average EF magnitude in these ROIs is plotted in Fig. 3, including the medial and inferior frontal gyri, hippocampi and thalamus of both hemispheres, as well as the SAC region. As is expected, the EF magnitude at ROIs was highly dependent upon the position of both electrodes, especially ROIs close to the cortical surface. For instance, the EF at the left frontal gyri differed substantially depending on whether the placement was bilateral or unilateral (where both electrodes were on the right side of the head): when there was a left electrode, the EF reached as high as $123.11 \mathrm{~V} / \mathrm{m}$ (e.g. SUB2 with BF), whereas it dropped to as low as $55.67 \mathrm{~V} / \mathrm{m}$ where both electrodes were on the right side (e.g. SUB2 with temporo-parietal-unilateral). On the other hand, the EF magnitude at the SAC regions depended on how close the electrodes were positioned to the midline, and thus the longitudinal fissure between two hemispheres: for SUB 1, the EF with midfrontooccipital, i.e. the midline placement, reached to $107.19 \mathrm{~V} / \mathrm{m}$, whereas it was only $48.81 \mathrm{~V} / \mathrm{m}$ with $\mathrm{BF}$. The $\mathrm{EF}$ at the hippocampus, located in the temporal lobe, had a significantly larger magnitude when an electrode was placed at the ipsilateral temporal position (e.g. BT, both temporo-parietal placements and LART for the right hippocampus) or along the midline (e.g. midfronto-occipital and all fronto-occipital placements). As for the thalamus, deep inside the brain tissue, this received the least amount of influence from the electrode position: the EF magnitude was moderately elevated when the electrodes were located close to the midline.

This dependency of EF magnitude on the electrode placement also appeared to be moderately influenced by inter-subject variability (e.g. head size and brain volume) or ROI size. The inter-subject differences among the three subjects are listed in Table 1. It can be observed in the table, that the largest difference in head sizes occurred between SUB1 and SUB2. Regarding the EF magnitude at the left hippocampus, the difference between BT and temporo-parietal-unilateral in SUB1 and SUB2 was $47.36 \mathrm{~V} / \mathrm{m}$ and $78.85 \mathrm{~V} / \mathrm{m}$ respectively, whereas that between temporo-parietalbilateral and supraorbito-parietal-bilateral in SUB1 and SUB2 was $10.98 \mathrm{~V} / \mathrm{m}$ and $1.34 \mathrm{~V} / \mathrm{m}$ respectively.

In order to examine the influence of ROI size on the EF magnitude, a sphere was overlayed with the SAC region, centred at the SAC with five different radii. Fig. $4 \mathrm{~b}$ demonstrates the resulting SAC volume within the spheres, and Fig. $4 a 1, a 2$ and $a 3$ respectively compare the average EF magnitude in regards to different SAC volume in SUB1, SUB2 and SUB3. As expected, the inter-subject variability also played a role in this situation. For SUB1, the changes among different SAC volume were small; however for SUB2 and SUB3, the changes were comparably larger for some placements - although the general shape of "curve of placements" remained mostly the same, the significance of inter-placement difference became smaller with an increasing SAC volume.

\section{Discussion}

We investigated the direct stimulation effects of ten alternative and novel ECT electrode placements relative to conventional electrode placements (i.e., BT, RUL, BF). Specifically, we examined stimulation effects in brain regions hypothesised to be associated with antidepressant efficacy as well as those considered to have a critical role in memory side-effects with the aim of identifying promising alternative methods for administering ECT for MDD.

\subsection{Alternative compared to conventional placements}

Fronto-parietal RUL ECT has been proposed as an alternative to temporo-parietal RUL that may have similar efficacy but less memory side effects $[29,30,25]$. Simulations support this hypothesis, finding similar EF in the SAC region, but less hippocampal stimulation, particularly in the right hemisphere. We investigated whether moving the anterior electrode more medially may confer additional advantages (the supraorbito-parietal-unilateral placement). Indeed, we found that the supraorbito-parietal-unilateral placement had similarly low EF in hippocampal regions as the fronto-parietal-unilateral placement, but higher EF in the SAC region, suggesting that moving the anterior electrode medially from the lateral forehead to a supraorbital position may further optimise ECT stimulation. Conversely, moving the posterior electrode to the other hemisphere (fronto-parietal-bilateral, supraorbito-parietal-bilateral), i.e. "bilateral" versions corresponding to fronto-parietal-unilateral and supraorbito-parietal-unilateral placements, resulted in higher EF in the left inferior and middle frontal gyri and hippocampi, i.e. areas likely to mediate greater cognitive side effects.

Moving the electrodes further apart while maintaining a unilateral placement (i.e. fronto-occipital) resulted in more diffuse stimulation, with greater stimulation of cognitive centres than standard RUL (temporo-parietal) ECT, i.e. may actually increase cognitive side effects. Stimulating down the midline (midfrontooccipital), in an attempt to strongly active the SAC region, resulted in the most diffuse stimulation of all placements. While EF was high in the SAC region, it was also relatively high in the frontal gyri and hippocampi, i.e. this placement is unlikely to improve on conventional BT and BF placements. LART may confer advantages to conventional BT ECT: our simulations showed stronger activation of the SAC region, with less hippocampal activation on the left.

\subsection{Role of interindividual differences in stimulation effects}

Unexpectedly, our simulations showed substantial differences in stimulation outcomes between the three subjects, suggesting 

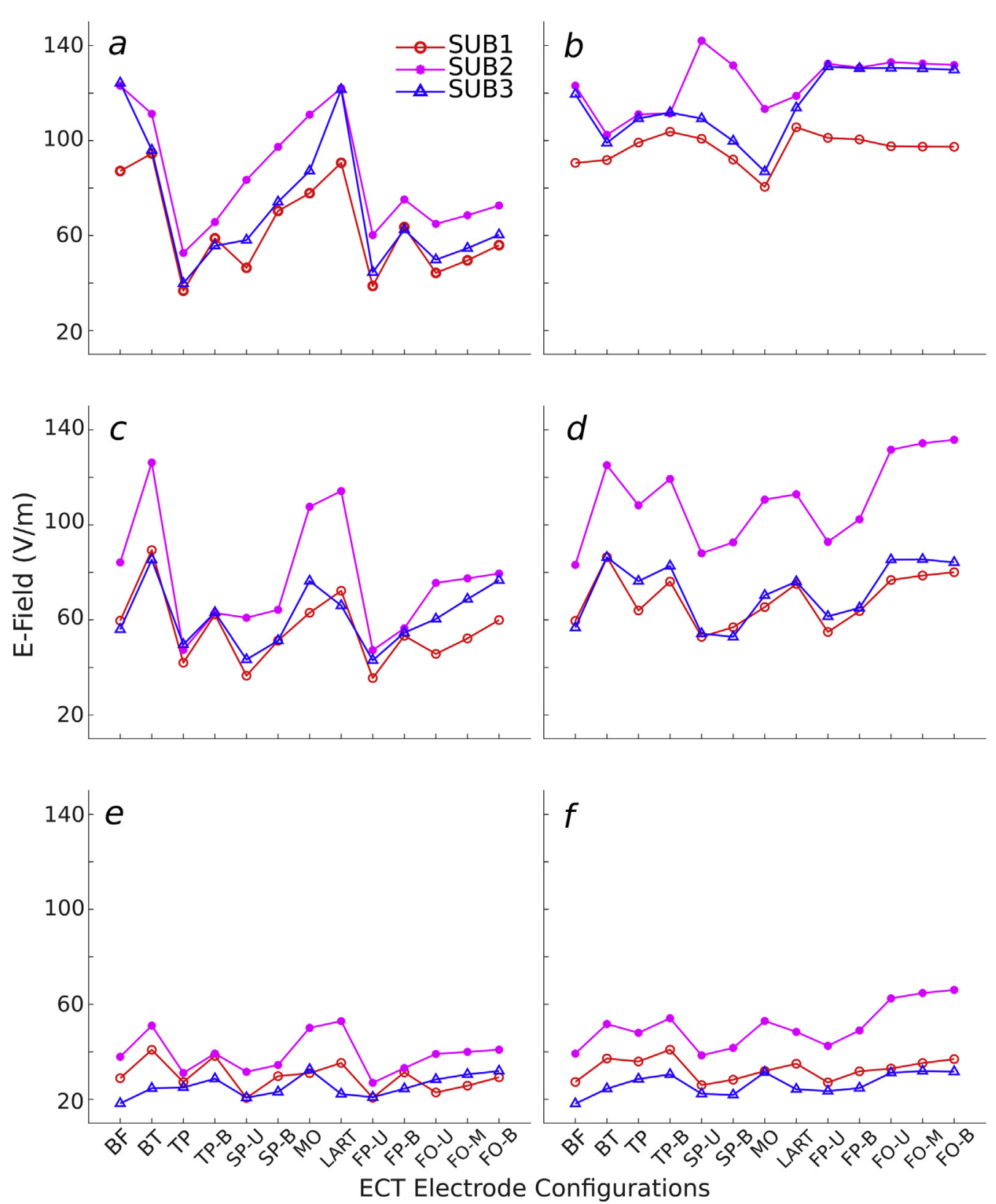

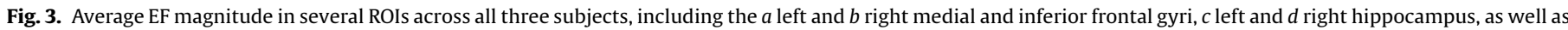

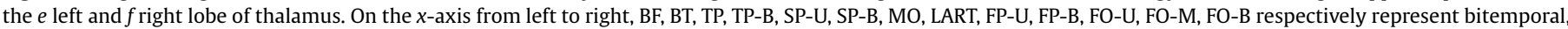

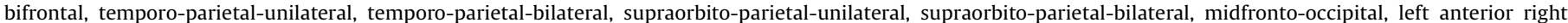
temporal, fronto-parietal-unilateral, fronto-parietal-bilateral, fronto-occipitalmedial, fronto-occipital-unilateral and fronto-occipital-bilateral.

that head size may be an important determinant of ECT clinical outcomes, even under the same electrode placement. For example, stimulation of the bilateral hippocampi, considered relevant to memory related side-effects, indicated that SUB2, who had the

\section{Table 1}

Inter-subject differences among the three subjects. The head size was characterised by the anterior-posterior (A-P) and left-right (L-R) straight-line distances. The brain volume included volumes of the cerebellum and brain stem.

\begin{tabular}{llll}
\hline & Head size & \\
\cline { 2 - 3 } & A-P distance $(\mathrm{mm})$ & L-R distance $(\mathrm{mm})$ & Brain volume $\left(\mathrm{mm}^{3}\right)$ \\
\hline SUB1 & 199.9 & 166.3 & 1293.8 \\
SUB2 & 185.4 & 139.9 & 863.4 \\
SUB3 & 202.8 & 161.1 & 1259.7 \\
\hline
\end{tabular}

smallest head, exhibited higher levels of stimulation $(>25 \mathrm{~V} / \mathrm{m})$ with conventional $\mathrm{BT}$ and $\mathrm{BF}$ electrode placements compared to the other two subjects. Head size (nasium to inion distance and head circumference) has previously been identified as a relevant factor affecting ECT convulsive threshold [48], with larger heads requiring greater stimulus charge, potentially due to greater resistance from the skull dimensions and/or greater diffusion of the current in regions important for seizure propagation. These findings together highlight the importance of individual seizure titration with ECT, as the use of fixed dosing methods in smaller heads may lead to disproportionately greater cognitive side-effects due to greater direct stimulation of memory-related regions. Future research into the association between ECT treatment dose, head size and memory side-effects is warranted to confirm this association. 


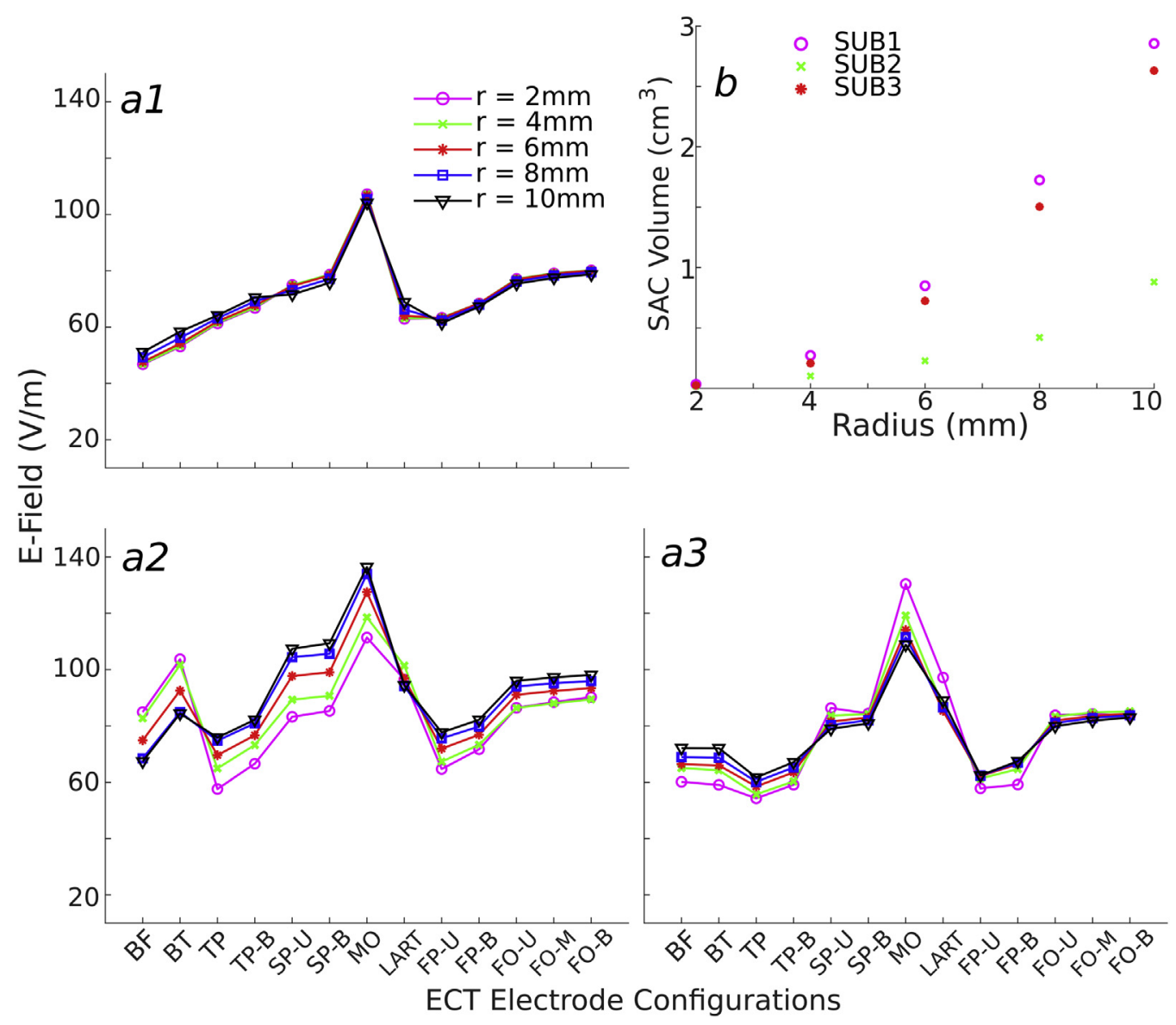

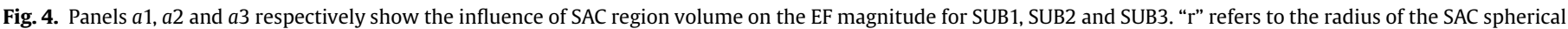

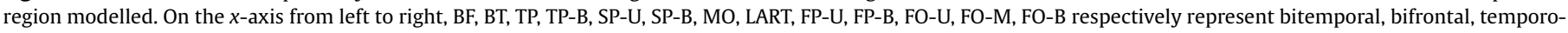

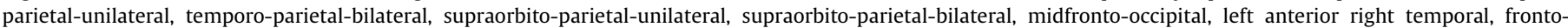

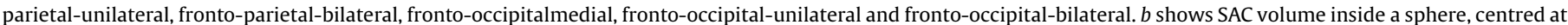
the centre of SAC, with five different radii.

\subsection{Limitations}

As discussed above, it is hypothesized that stimulation at the SAC region is important for the efficacy of ECT, and that minimal stimulation of known memory-related regions would reduce the cognitive side effects of ECT. These hypotheses are based on associated sources of information about the roles of these brain regions. However, to date a direct link between stimulation or avoidance of these regions, and outcomes of ECT, is yet to be proven. It is also possible that minimising stimulation in some regions may be important for efficacy and increased stimulation in other regions may be important for improved cognitive outcomes, but this is speculative. The simulations were done in three individual subjects, who covered a range of head sizes, demonstrating the importance of inter-individual differences, and it is likely that a larger number of subjects would further demonstrate the principle of inter-individual differences. Future research utilising modelling and clinical outcomes will be important to confirm hypotheses regarding stimulation and clinical effects.

\subsection{Conclusions}

Compared to conventional RUL ECT using a temporo-parietal placement, fronto-parietal and supraorbito-parietal RUL also robustly stimulated brain regions considered important for efficacy, while sparing regions related to cognitive functions, and may be a preferrable approach to the currently used placement for RUL ECT. Clinical trials comparing these approaches would be warranted. The simulations also found considerable inter-individual differences in stimulation effects due to factors such as head size.

\section{Acknowledgements}

The authors would like to thank Prof. Caroline Rae from Neuroscience Research Australia for providing the structural MRI of SUB1 and SUB2, Dr. Elizabeth Tancred from the University of New South Wales for her expertise in the neuroanatomy, and Prof. Marom Bikson and Mr. Dennis Truong from the City College of New York for their contributions in reconstructing SUB3. Dr. Bai was the holder of a Humboldt Research Fellowship for Postdoctoral Researchers from Alexander von Humboldt Foundation during the study.

\section{References}

[1] Kellner CH, Knapp R, Husain MM, Rasmussen K, Sampson S, Cullum M, et al. Bifrontal, bitemporal and right unilateral electrode placement in ECT: randomised trial. Br J Psychiatry 2010;196(3):226-34.

[2] Sackeim HA, Prudic J, Devanand DP, Kiersky JE, Fitzsimons L, Moody BJ, et al. Effects of stimulus intensity and electrode placement on the efficacy and cognitive effects of electroconvulsive therapy. N Engl J Med 1993;328 (12):839-46.

[3] Sackeim HA, Prudic J, Devanand DP, Nobler MS, Lisanby SH, Peyser S, et al. A prospective, randomized, double-blind comparison of bilateral and right 
unilateral electroconvulsive therapy at different stimulus intensities. Arch Gen Psychiatry 2000;57(5):425-34.

[4] D'Elia G. Unilateral electroconvulsive therapy. Acta Neurol Scand Suppl 1970;215:1.

[5] Sackeim HA, Prudic J, Nobler MS, Fitzsimons L, Lisanby SH, Payne N, et al. Effects of pulse width and electrode placement on the efficacy and cognitive effects of electroconvulsive therapy. Brain Stimul 2008;1(2):71-83.

[6] McCall WV, Reboussin DM, Weiner RD, Sackeim HA. Titrated moderately suprathreshold vs fixed high-dose right unilateral electroconvulsive therapy: acute antidepressant and cognitive effects. Arch Gen Psychiatry 2000;57 (5):438-44.

[7] Abrams R, Fink M. Clinical experiences with multiple electroconvulsive treatments. Compr Psychiatry 1972;13(2):115-21.

[8] Abrams R, Taylor MA. Anterior bifrontal ECT: a clinical trial. Br J Psychiatry 1973;122(570):587-90.

[9] Bailine SH, Rifkin A, Kayne E, Selzer JA, Vital-Herne J, Blieka M, et al. Comparison of bifrontal and bitemporal ECT for major depression. Am J Psychiatry 2000;157(1):121-3.

[10] Dunne RA, McLoughlin DM. Systematic review and meta-analysis of bifrontal electroconvulsive therapy versus bilateral and unilateral electroconvulsive therapy in depression. World J Biol Psychiatry 2012;13(4):248-58.

[11] Lawson JS, Inglis J, Delva NJ, Rodenburg M, Waldron JJ, Letemendia FJJ. Electrode placement in ECT: cognitive effects. Psychol Med 1990;20(02):335-44.

[12] Letemendia FJJ, Delva NJ, Rodenburg M, Lawson JS, Inglis J, Waldron JJ, et al. Therapeutic advantage of bifrontal electrode placement in ECT. Psychol Med 1993;23(02):349-60.

[13] Ranjkesh F, Barekatain M, Akuchakian S. Bifrontal versus right unilateral and bitemporal electroconvulsive therapy in major depressive disorder. J ECT 2005;21(4):207-10.

[14] Bai S, Loo C, Dokos S. Electroconvulsive therapy simulations using an anatomically-realistic head model. Conf Proc IEEE Eng Med Biol Soc, 2011 2011;5484-7.

[15] Bai S, Loo C, Al Abed A, Dokos S. A computational model of direct brain excitation induced by electroconvulsive therapy: comparison among three conventional electrode placements. Brain Stimulat 2012;5(3):408-21.

[16] Bai S, Loo C, Dokos S. Effects of electroconvulsive therapy stimulus pulsewidth and amplitude computed with an anatomically-realistic head model. Conf Proc IEEE Eng Med Biol Soc, 2012. . p. 2559-62.

[17] Bai S, Loo C, Lovell NH, Dokos S. Comparison of three right-unilateral electroconvulsive therapy montages. Conf Proc IEEE Eng Med Biol Soc, 2013 2013;819-22.

[18] Bai S, Gálvez V, Dokos S, Martin D, Bikson M, Loo C. Computational models of bitemporal, bifrontal and right unilateral ECT predict differential stimulation of brain regions associated with efficacy and cognitive side effects. Eur Psychiatry 2017;41:21-9.

[19] Lee WH, Deng ZD, Kim TS, Laine AF, Lisanby SH, Peterchev AV. Regional electric field induced by electroconvulsive therapy in a realistic finite element head model: influence of white matter anisotropic conductivity. Neuroimage 2012;59(3):2110-23.

[20] Lee WH, Lisanby SH, Laine AF, Peterchev AV. Comparison of electric field strength and spatial distribution of electroconvulsive therapy and magnetic seizure therapy in a realistic human head model. Eur Psychiatry 2016;36:55-64.

[21] McNally KA, Blumenfeld $\mathrm{H}$. Focal network involvement in generalized seizures: new insights from electroconvulsive therapy. Epilepsy Behav 2004;5(1):3-12.

[22] UK ECT Review. Efficacy and safety of electroconvulsive therapy in depressive disorders: a systematic review and meta-analysis. Lancet 2003;361 (9360):799-808.

[23] Regenold WT, Noorani RJ, Piez D, Patel P. Nonconvulsive electrotherapy for treatment resistant unipolar and bipolar major depressive disorder: a proofof-concept trial. Brain Stimul 2015;8(5):855-61.

[24] Swartz C. Asymmetric bilateral right frontotemporal left frontal stimulus electrode placement for electroconvulsive therapy. Neuropsychobiology 1994;29(4):174-8.
[25] Loo C, Bai S, Martin McLinneuro D, Gálvez V, Dokos S. Revisiting frontoparietal montage in electroconvulsive therapy: clinical observations and computer modeling: a future treatment option for unilateral electroconvulsive therapy. J ECT 2015:31(1):e7-e13.

[26] Spellman T, Peterchev AV, Lisanby SH. Focal electrically administered seizure therapy: a novel form of $\{\mathrm{ECT}\}$ illustrates the roles of current directionality, polarity, and electrode configuration in seizure induction. Neuropsychopharmacology 2009;34(8):2002-10.

[27] Swartz CM, Evans CM. Beyond bitemporal and right unilateral electrode placements. Psychiatric Annals 1996;26(11):705-8.

[28] Swartz CM. Physiological response to ECT stimulus dose. Psychiatry Res 2000;97(2-3):229-35.

[29] D'Elia G, Widepalm K. Comparison of frontoparietal and temporoparietal unilateral electroconvulsive therapy. Acta Psychiatr Scand 1974:50(2):225-32.

[30] D'Elia G, Lorentzson S, Raotma H, Widepalm K. Comparison of unilateral dominant and non-dominant $\{\mathrm{ECT}\}$ on verbal and non-verbal memory. Acta Psychiatr Scand 1976;53(2):85-94.

[31] Nahas Z, Short B, Burns C, Archer M, Schmidt M, Prudic J, et al. A feasibility study of a new method for electrically producing seizures in man: focal electrically administered seizure therapy [FEAST]. Brain Stimulat 2013;6 (3):403-8.

[32] Sahlem GL, Short EB, Kerns S, Snipes J, DeVries W, Fox JB, et al. Expanded safety and efficacy data for a new method of performing electroconvulsive therapy. J ECT 2016;32(3):197-203.

[33] Anderson RJ, Frye MA, Abulseoud OA, Lee KH, McGillivray JA, Berk M, et al. Deep brain stimulation for treatment-resistant depression: efficacy, safety and mechanisms of action. Neurosci Biobehav Rev 2012;36(8):1920-33.

[34] Drevets WC, Price JL, Furey ML. Brain structural and functional abnormalities in mood disorders: implications for neurocircuitry models of depression. Brain Struct Funct 2008;213(1-2):93-118.

[35] Mayberg HS, Lozano AM, Voon V, McNeely HE, Seminowicz D, Hamani C, et al. Deep brain stimulation for treatment-resistant depression. Neuron 2005;45 (5):651-60

[36] McCormick LM, Yamada T, Yeh M, Brumm MC, Thatcher R. Antipsychotic effect of electroconvulsive therapy is related to normalization of subgenual cingulate theta activity in psychotic depression. J Psychiatr Res 2009;43(5):553-60.

[37] Semkovska M, McLoughlin DM. Objective cognitive performance associated with electroconvulsive therapy for depression: a systematic review and metaanalysis. Biol Psychiatry 2010:68(6):568-77.

[38] Sackeim HA. Autobiographical memory and electroconvulsive therapy. J ECT 2014;30(3):177-86.

[39] Squire L. Mechanisms of memory. Science 1986;232(4758):1612-9.

[40] Nadel L, Hardt O. Update on memory systems and processes. Neuropsychopharmacology 2011;36(1):251-73.

[41] Daselaar SM, Veltman DJ, Rombouts SARB, Raaijmakers JGW, Jonker C Neuroanatomical correlates of episodic encoding and retrieval in young and elderly subjects. Brain 2003;126(1):43-56.

[42] Nyberg L. Mapping episodic memory. Behav Brain Res 1998;90(2):107-14.

[43] Fridriksson J, den Ouden DB, Hillis AE, Hickok G, Rorden C, Basilakos A, et al. Anatomy of aphasia revisited. Brain 2018;141(3):848-62.

[44] Kreisler A, Godefroy O, Delmaire C, Debachy B, Leclercq M, Pruvo JP, et al. The anatomy of aphasia revisited. Neurology 2000;54(5):1117-23.

[45] Bai S, Dokos S, Ho KA, Loo C. A computational modelling study of transcranial direct current stimulation montages used in depression. Neuroimage 2014;87:332-44.

[46] Ho KA, Bai S, Martin D, Alonzo A, Dokos S, Puras P, et al. A pilot study of alternative transcranial direct current stimulation electrode montages for the treatment of major depression. J Affect Disord 2014;167:.

[47] Bikson M, Dmochowski J, Rahman A. The quasi-uniform assumption in animal and computational models of non-invasive electrical stimulation. Brain Stimulat 2013;6(4):704-5.

[48] McCall W, Shelp FE, Weiner RD, Austin S, Norris J. Convulsive threshold differences in right unilateral and bilateral ECT. Biol Psychiatry 1993;34 (9):606-11. 